\title{
Crucial role of the pentose phosphate pathway in malignant tumors (Review)
}

\author{
LIN JIN $^{1,2}$ and YANHONG ZHOU ${ }^{1,2}$ \\ ${ }^{1}$ The Key Laboratory of Carcinogenesis of The Chinese Ministry of Health, Xiangya Hospital; \\ ${ }^{2}$ The Key Laboratory of Carcinogenesis and Cancer Invasion of The Chinese Ministry of Education, \\ Cancer Research Institute, Central South University, Changsha, Hunan 410078, P.R. China
}

Received May 8, 2018; Accepted January 4, 2019

DOI: $10.3892 / 01.2019 .10112$

\begin{abstract}
Interest in cancer metabolism has increased in recent years. The pentose phosphate pathway (PPP) is a major glucose catabolism pathway that directs glucose flux to its oxidative branch and leads to the production of a reduced form of nicotinamide adenine dinucleotide phosphate and nucleic acid. The PPP serves a vital role in regulating cancer cell growth and involves many enzymes. The aim of the present review was to describe the recent discoveries associated with the deregulatory mechanisms of the PPP and glycolysis in malignant tumors, particularly in hepatocellular carcinoma, breast and lung cancer.
\end{abstract}

Correspondence to: Professor Yanhong Zhou, The Key Laboratory of Carcinogenesis of The Chinese Ministry of Health, Xiangya Hospital, Central South University, 110 Xiangya Road, Changsha, Hunan 410078, P.R. China

E-mail: zhouyanhong@csu.edu.cn

Abbreviations: 3-PG, 3-phosphoglycerate; 6PGD, 6-phosphogluconate dehydrogenase; AML, acute myeloid leukemia; ATP, adenosine 5'-triphosphate; BAG, Bcl-2-associated athanogene; EMT, epithelial-mesenchymal transition; F6P, fructose 6-phosphate; G3P, glyceraldehyde 3-phosphate; G6PD, glucose 6-phosphatedehydrogenase; HCC, hepatocellular carcinoma; HIF-1, hypoxia-inducible factor 1; HK, hexokinase; NADPH, nicotinamide-adenine dinucleotide phosphate; NRF2, nuclear factor, erythroid 2-like 2; NSCLC, non-small cell lung carcinoma; PFK1, phosphofructokinase-1; PFKFB3, 6-phosphofructo-2-kinase/fructose-2,6-bisphosphatase 3; PFKP, PFK1, platelet isoform; PGAM1, phosphoglycerate mutase 1; PK, pyruvate kinase; PKM2, pyruvate kinase M2 isoform; Plk1, Polo-like kinase 1; PPP, pentose phosphate pathway; PTEN, phosphatase and tensin homolog; R5P, ribose-5-phosphate; RPE, ribulose-5-phosphate epimerase; RPI, ribose-5-phosphate isomerase; RPIA, ribose-5-phosphate isomerase A; Ru5P, ribulose-5-phosphate; STAT3, signal transduction and activator of transcription 3; TALDO, transaldolase; TKT, transketolase; TKTL1, transketolase-like 1; Xu5P, xylulose-5-phosphate

Key words: pentose phosphate pathway, glycolysis, hepatocellular carcinoma, breast cancer

\section{Contents}

1. Introduction

2. Glucose in the PPP

3. Glucose breakdown through glycolysis in the PPP

4. PPP in malignant tumors

5. Perspectives

\section{Introduction}

Metabolic adaptations are closely associated with alterations in cellular behavior. In the past 20 years, there has been a growing interest in cancer metabolism, particularly on glucose metabolism (1). Cancer cells are able to reprogram their energy metabolism to meet the increased biogenetic demands required for their rapid and uncontrolled growth (2). Cells from normal tissues mainly generate adenosine 5'-triphosphate (ATP) through the mitochondrial oxidative phosphorylation. In these cells, glucose is transformed to pyruvate through glycolysis, and most pyruvate enters mitochondrial oxidative metabolism for efficient energy generation (3). However, most cancer cells consume glucose through glycolysis, even in the presence of sufficient oxygen; this phenomenon is called the Warburg effect, which leads to the production of pyruvate and lactate as final metabolites (4). This enhanced aerobic glycolysis allows cancer cells to better proliferate by generating sufficient amounts of ATP and other biomolecules, including nucleotides, amino acids and fatty acids (5).

The pentose phosphate pathway (PPP), also known as the phosphogluconate pathway or the hexose monophosphate shunt, is a metabolic pathway parallel to glycolysis, and represents the first committed step of glucose metabolism (6). The PPP serves a pivotal role in supporting cancer cell survival and growth by generating pentose phosphate for nucleic acid synthesis and providing nicotinamide-adenine dinucleotide phosphate (NADPH), which is needed for fatty acid synthesis and cell survival under stress conditions (7). Previous studies indicate that PPP flux can be directly or indirectly modulated in cancer cells, in order to improve cell survival and proliferation $(2,7)$. Therefore, the regulatory network of PPP flux represents an important metabolic adaptation in a number of environmental contexts in human malignancies, including cancer. 


\section{Glucose in the PPP}

The PPP occurs in the cytosol and comprises two irreversible oxidative reactions followed by a series of reversible interconversions (Fig. 1). The PPP is thus divided into two biochemical branches: An oxidative and a non-oxidative branch. The oxidative branch converts glucose 6-phosphate (G6P) into ribulose-5-phosphate (Ru5P), $\mathrm{CO}_{2}$ and NADPH (8). NADPH is vital to maintain the reduction-oxidation (redox) balance under stress conditions and allows cells to proliferate rapidly (9). The non-oxidative branch yields the glycolytic intermediates fructose 6-phosphate (F6P), glyceraldehyde 3 -phosphate (G3P) and sedoheptulose sugars, resulting in the production of sugar phosphate precursors for amino acid synthesis and ribose-5-phosphate (R5P), which is essential for nucleic acid synthesis (10).

Role of glucose 6-phosphate dehydrogenase (G6PD) in the $P P P$. The PPP is primarily regulated during the G6PD reaction. G6PD catalyzes the irreversible oxidation of G6P into 6-phosphogluconolactone in a rate-limiting step; the first molecule of NADPH is generated during this reaction (11). G6PD acts as a 'gatekeeper' of this pathway and is therefore the rate-limiting enzyme in the PPP. Subsequently, G6PD activity not only determines the flux partitioning between glycolysis and PPP, but also reflects the oxidative PPP flux (12). G6PD is overexpressed in cancer cells, and Ju et al (13) demonstrated that the elevated expression of G6PD is predictive of poor survival of patients with cancer, indicating that G6PD may serve a vital role in tumorigenesis. There are two cellular isomers of G6PD, a dimer and a tetramer; the dimer stability has been demonstrated to have an important role in vivo (14). High $\mathrm{pH}$ and ionic strength are beneficial for the dimer synthesis, whereas low $\mathrm{pH}$ generates a shift toward the tetramer synthesis (15).

The tumor suppressor p53 binds to G6PD and inhibits the formation of the active dimer and suppresses NADPH production, glucose consumption and biosynthesis, which results in inhibition of the PPP (16). Polo-like kinase 1 (Plk1) is a key regulator of cell mitosis and enhances PPP flux and macromolecule biosynthesis through the direct phosphorylation of G6PD to promote the formation of G6PD active dimer. This is an essential feature of Plk1 as a promoter of cancer cell cycle progression and growth (17). In addition, glycosylation activates G6PD activity, and modification of G6PD with an $\mathrm{O}$-linked $\beta$-N-acetylglucosamine sugar increases the glucose flux to the PPP (18). Mammalian target of rapamycin complex 1 upregulates the transcriptional and the post-transcriptional expression of G6PD to activate PPP (19). p21-activated kinase 4 increases G6PD activity by enhancing Mdm2-mediated p53 ubiquitination and degradation (20). Furthermore, suppression of G6PD lowers glutathione levels, decreases NADPH production, reduces the capacity to scavenge reactive oxygen species (ROS) and enhances the oxaliplatin-induced apoptosis through ROS-mediated damage in vitro (13). These results indicate that G6PD may be a potential prognostic biomarker and represent a promising target in cancer therapy.

Role of 6-phosphogluconate dehydrogenase (6PGD) in the $P P P$. The 6-phosphogluconolactone hydrolase irreversibly hydrolyzes 6-phosphogluconolactone into 6-phosphogluconate
(6PG). 6PG is then oxidatively decarboxylated by 6PGD, leading to the synthesis of Ru5P, $\mathrm{CO}_{2}$ and a second molecule of NADPH. Upregulation of 6PGD activity has been identified in various types of cancer, including breast, acute myeloid leukemia (AML), ovarian and lung cancers (21-23).

The enzyme 6PGD is commonly activated in human cancer cells after lysine acetylation, which promotes $\mathrm{NADP}^{+}$ binding to 6PGD and the formation of active dimers of 6PGD (24). In this pathway, activated 6PGD enhances the oxidative phase of PPP, and nucleotide or RNA biosynthesis. This reaction serves a role in maintaining intracellular Ru5P at a physiological level that is sufficient to fulfill the metabolic requirements of rapidly growing cancer cells (25). In addition, 3-phosphoglycerate (3-PG) directly binds to the active site of 6PGD and competes with its substrate, 6PG, to inhibit 6PGD. Furthermore, the glycolytic enzyme phosphoglycerate mutase 1 (PGAM1) controls intracellular levels of 3-PG (26). A recent study reported that attenuation of PGAM1 results in abnormal accumulation of 3-PG, which inhibits 6PGD and subsequently suppresses the oxidative PPP and anabolic biosynthesis. Malic enzyme forms a physiological hetero-oligomer with 6PGD, which increases 6PGD activity (27).

Roles of ribose-5-phosphate isomerase (RPI) and ribulose-5-phosphate epimerase (RPE) in the PPP. The enzyme RPI converts Ru5P into R5P, and the enzyme RPE converts Ru5P into xylulose-5-phosphate (Xu5P). It has been demonstrated that ribose-5-phosphate isomerase A (RPIA) regulates cancer growth and tumorigenesis (28). In addition, RPIA is significantly overexpressed in colorectal cancer and hepatocellular carcinoma (HCC) $(29,30)$. RPIA also activates $\beta$-catenin by entering the nucleus to form a complex with adenomatous polyposis coli and $\beta$-catenin, thus modulating cell proliferation and oncogenicity (29).

Roles of transketolase (TKT) and transaldolase (TALDO) in the PPP. TKT and TALDO are two enzymes that convert R5P and Xu5P, and the gluconeogenetic intermediates G3P and F6P. TKT and TALDO are responsible for complex interconversion reactions within the non-oxidative PPP (10). TKT converts excess R5P into G3P and F6P through a number of reactions, $\mathrm{G} 3 \mathrm{P}$ is metabolized alongside further steps of glycolysis, and F6P is converted into G6P that re-enters the oxidative PPP to generate additional NADPH (31). Elevated TKT expression levels were reported in lung cancer cells, breast cancer cells and prostate cancer cells $(21,22)$.

TKT expression is closely regulated by the nuclear factor, erythroid 2-like 2 (NRF2)/Kelch-like ECH-associated protein 1/BTB and CNC homolog 1 oxidative stress sensor pathway in various types of cancer (32). For example, exposure to ultraviolet A increases cancer proliferation by upregulating intracellular concentrations of TKT in melanoma (33). In addition, fructose stimulates TKT activity and is preferentially used over glucose to generate nucleic acids via the non-oxidative PPP (34). Higher vertebrates obtain transketolase-like 1 (TKTL1) by genome duplication and exon skipping $(35,36)$. TKTL1 upregulation is a general phenomenon in epithelial malignancies, ocular adnexal tumors, malignant pleural effusion and other types of cancer (37-39). TKTL1 is therefore considered a novel tumor marker and a potential good target in cancer treatment (40). 


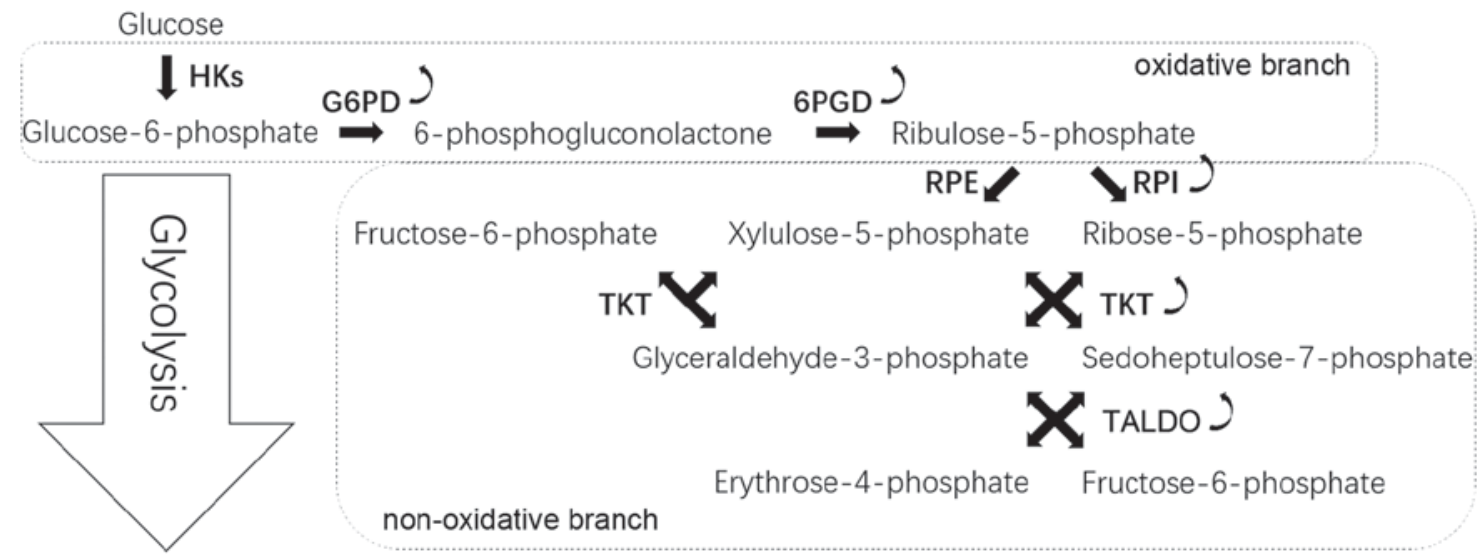

Figure 1. PPP. G6PD converts glucose-6-phosphate into 6-phosphogluconolactone. 6-Phosphogluconolactone then converted to Ru5P by 6PGD. Ru5P undergoes isomerization by RPI or RPE to generate ribose-5-phosphate or xylulose-5-phosphate, respectively. In the non-oxidative reactions of PPP, TKT and TALDO are responsible for relatively complex interconversion reactions. Curved arrows indicate that G6PD, 6PGD, RPI, TKT and TALDO are overexpressed in cancer cells. 6PGD, 6-phosphogluconate dehydrogenase; G6PD, glucose 6-phosphate dehydrogenase; PPP, pentose phosphate pathway; RPE, ribulose-5-phosphate epimerase; RPI, ribose 5-phosphate isomerase; Ru5P, ribulose-5-phosphate; TALDO, transaldolase; TKT, transketolase.

TALDO catalyzes the reversible transfer of a three-carbon unit between various sugar phosphates (from ketose to aldose sugar phosphates) (10). A previous study has revealed that TALDO is significantly overexpressed in gastric adenocarcinoma (41). Furthermore, its expression is associated with metastatic behavior in HCC (42). In addition, a combination of arginine and ascorbic acid decreases intracellular NADPH levels by reducing TALDO activity in the PPP (43).

\section{Glucose breakdown through glycolysis influencing PPP}

Numerous regulatory pathways for tumor cells exist within the PPP, and most reactions in glycolysis are crucial to maintain tumor cell function. Since PPP and glycolysis are metabolically linked for sharing the common intermediate G6P, the increased glycolysis during reperfusion concomitantly led to decreased PPP rate (44). The conversion of glucose to pyruvate occurs in two stages (Fig. 2). In the first stage, phosphorylated forms of pyruvate intermediates are synthesized, leading to ATP synthesis. Hexokinase (HK) phosphorylates glucose into G6P, and phosphofructokinase-1 (PFK1) catalyzes the conversion of F6P to fructose 1,6-bisphosphate. In the subsequent stage, ATP is generated by substrate-level phosphorylation and metabolism of glucose. The final step of glycolysis is catalyzed by the pyruvate kinase (PK) enzyme that leads to the synthesis of pyruvate and ATP. In cancer cells, the glycolytic reaction generates a 'bottleneck' effect by increasing the upstream part of the glycolytic flux up to PK and decreasing the glycolytic flux from PK downward (45).

Role of HK in the conversion of glucose by glycolysis in the $P P P$. HK catalyzes glucose phosphorylation, which is one of the regulatory reactions of glycolysis. To maintain the Warburg effect, cancer cells upregulate HK. Four isoforms of HK exist (HK1-HK4). HK2, which may be in a soluble form in the cytoplasm or bound to the mitochondrial outer membrane, has a glucose affinity 100-fold higher than HK1, HK3 and HK4 (46). In addition, the expression of HK1 may be sufficient for normal cell metabolism. However, the accelerated anabolic metabolism in cancer cells demands a robust
HK activity. Therefore, the induction of HK2 expression is required. Overall, HK2 is elevated in cancer cells, promotes glycolysis and inhibits mitochondrial-mediated apoptosis (47).

The induction of HK2 expression by oncogenic Ras is crucial for accelerated ribonucleotide synthesis (48). Bcl-2-associated athanogene (BAG)-3, a member of the BAG cochaperone family that comprises six BAGs (BAG1-BAG6), increases HK2 expression by interacting with HK2 mRNA (49). Hypoxia-inducible factor (HIF)-1 $\alpha$ induces the expression of the glycolytic enzyme HK2. The sustained expression of the oncogene forms of the human papillomavirus E6 and E7 is vital to maintain HK2 expression levels by upregulating the pro-oncogene MYC and downregulating microRNA (miR)-143-3p (50). In AML, an internal tandem duplication mutation in the Fms-like tyrosine kinase 3 gene upregulates the level of mitochondrial HK2, causing a significant increase in aerobic glycolysis; therefore, leukemic cells become highly dependent on glycolysis, which increases their sensitivity to the pharmacological inhibition of glycolytic activity (51). In addition, the histone-lysine $\mathrm{N}$-methyltransferase NSD2 is recruited to and methylates HK2 promoters (52). NSD2-driven tamoxifen-resistant cancers exhibit an enhanced PPP activity, elevated NADPH production and reduced ROS levels. For example, treatment of ovarian cancer xenografted mice with the HK2 inhibitor 3-bromopyruvate attenuates tumor growth and confers a survival advantage (53).

Role of phosphofructokinase in the conversion of glucose by glycolysis in the PPP. PFK1 irreversibly phosphorylates F6P into fructose-1,6-bisphosphate. This reaction is a crucial and a rate-limiting step in glycolysis. It has been demonstrated that PFK1 activity is increased in cancer cell lines, and expression of PFK1 is upregulated in breast and liver cancers $(54,55)$. In addition, PFK1 is regulated by ATP and F6P substrates (56).

In response to hypoxia, O-GlcNAcylation suppresses PFK1 activity and redirects glucose towards the PPP, which provides an advantage for cancer cell growth (57). A Krüppel-associated box-type zinc-finger protein named p53 inhibitor of TIGAR activation (PITA) is a selective regulator of p53, and PITA transgenic mice exhibit increased PFK1 activity and elevated 


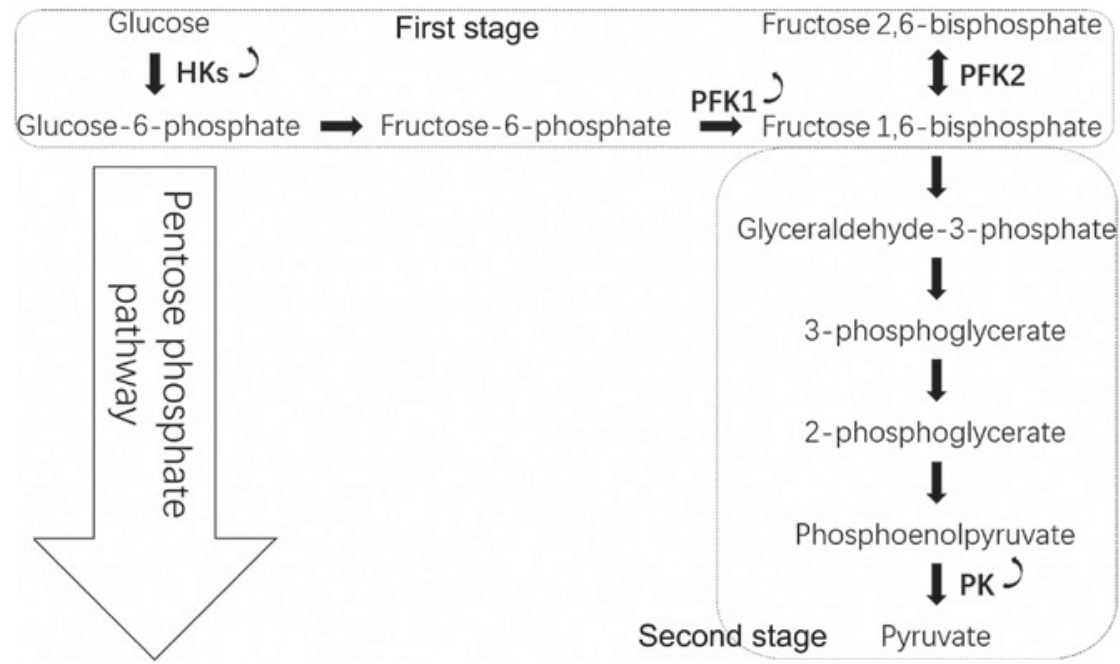

Figure 2. Glycolysis. Glycolysis consists of two stages, the first stage consuming ATP and the second stage generating ATP. There are three crucial reactions in tumor cells. First, HK catalyzes the conversion of glucose to glucose-6-phosphate. Second, fructose-6-phosphate is converted into fructose 1,6-bisphosphate by PFK1, and fructose 1,6-bisphosphate is converted into fructose 2,6-bisphosphate by PFK2. Third, phosphoenolpyruvate is converted into pyruvate by PK. Curved arrows indicate that HK, PFK1 and PK are overexpressed in cancer cells. HK, hexokinase; PK, pyruvate kinase; PFK, phosphofructokinase.

glycolytic rate (58). The PFK1 platelet isoform (PFKP), the predominant PFK1 isoform, is overexpressed in human glioblastoma cells and promotes aerobic glycolysis and brain cancer cell proliferation (59). In addition, the loss of phosphatase and tensin homolog (PTEN) and activation of epidermal growth factor receptor (EGFR)-dependent phosphoinositide 3-kinase cause AKT activation, which in turn increases PFKP stability (59). In leukemic cells, the cyclin D3-cyclin dependent kinase 6 (CDK6) phosphorylates PFKP and suppresses its activity (60), thus shifting the glucose-derived carbon into the PPP. Through this mechanism, cyclin D3-CDK6 enhances NADPH production to neutralize ROS. Snaill, which is a key transcriptional repressor of epithelial-mesenchymal transition (EMT), represses PFKP, leading to the glucose flux switch to PPP and the generation of NADPH (61). In addition, heme oxygenase-1/carbon monoxide reduces methylation of 6-phosphofructo-2-kinase/fructose-2,6-bisphosphatase 3 (PFKFB3) in cancer cells, thus redirecting glucose from the glycolysis pathway to the PPP, ensuring cancer cell resistance against oxidative stress (62). The dynamic regulation of PFKP enhances the survival of cancer cells undergoing metabolic stress and therefore increases their ability to metastasize in vivo.

Role of PK in the conversion of glucose by glycolysis in the $P P P$. PK converts phosphoenolpyruvate into pyruvate during the third irreversible reaction of glycolysis; thus, PK serves an important role in the control of metabolism in cancer cells. The ratio between the active and inactive forms of PK in cancer cells determines whether glucose is used for OXPHOS or for PPP to support cell growth (63). Low pyruvate kinase activity increases glucose influx into PPP for biosynthesis while high pyruvate kinase activity increases OXPHOS and decreases glucose influx into PPP (64). PK possesses two isoforms generated by alternative splicing of PK named M1 and M2, of which expressions are location and time dependent: The pyruvate kinase M2 isoform (PKM2) is preferentially expressed in cancer cells, where complex regulation of its activity is essential for the control of cellular metabolism (65).
Upon glucose starvation, cellular levels of succinylaminoimidazole-carboxamide riboside, an intermediate of the de novo purine nucleotide synthesis pathway, are increased. This leads to the stimulation of PKM2 activity in cancer cells, which alters cellular energy level, glucose uptake and lactate generation (66). Following EGFR activation, PKM2 binds and phosphorylates histone $\mathrm{H} 3$ at T11. PKM2-dependent histone $\mathrm{H} 3$ modification contributes to EGF-induced cyclin D1 and c-MYC expression, tumor cell proliferation, cell cycle progression and brain tumorigenesis (67). In human lung cancer cells, the marked increase in intracellular ROS leads to the inhibition of the glycolytic enzyme PKM2 by oxidation of Cys358, which requires the transfer of glucose flux into the PPP, stimulating redox potential and ROS detoxification (68). In addition, PKM2 gene transcription is activated by HIF- 1 by direct interaction with the HIF-1 $\alpha$ subunit (69). Serine binds to and activates human PKM2, and the PKM2 activity in cells after depletion of serine is reduced. This reduction in PKM2 activity switches the cells into a fuel-saving mode in which more pyruvate is transferred to mitochondria to support cell proliferation (70).

\section{PPP in malignant tumors}

HCC is one of the most common cancers worldwide (71). Breast cancer is the second most common cancer in the world, with 1.7 million new cases diagnosed annually (72). Lung cancer is the leading cause of cancer-associated mortality worldwide (73). One of the main features of these three malignancies is the alteration of glucose metabolism. Improved understanding of this metabolic alteration may therefore serve to optimize strategies for the prevention, early diagnosis and treatment of HCC, breast and lung cancer. In addition, a thorough understanding of cancer cell metabolism may provide potential novel therapeutic strategies for various types of cancer.

$P P P$ in HCC. Elevated expression of G6PD is associated with $\mathrm{HCC}$ metastases and poor prognosis of patients with HCC, and G6PD knockdown inhibits the proliferation, migration 
and invasion of HCC cell lines in vitro (74). In addition, G6PD promotes $\mathrm{HCC}$ cell migration and invasion by activating the signal transduction and activator of transcription 3 (STAT3) pathway to induce EMT (74). The transcription factor NRF2 is required for G6PD induction, and miR-1 is involved in its activation (75). BAG directly interacts with G6PD to suppress the PPP flux, DNA synthesis and HCC cell growth (76). Furthermore, PTEN binds to G6PD to prevent formation of the active G6PD dimer, which subsequently inhibits the PPP. However, the AKT coactivator T cell leukemia/lymphoma protein IA promotes G6PD activity and increases G6PD pre-mRNA splicing and protein expression (77). Inhibitor of differentiation and DNA binding-1 (ID1), regulates c-MYC through Wnt/ $\beta$-catenin pathway activation to promote G6PD promoter transcription and activate the $\operatorname{PPP}(78)$, which confers to HCC cells an oxaliplatin chemoresistance (79). In addition, ID1 activates the PPP to increase NADPH production and reduce intracellular ROS levels, thus promoting chemotherapy resistance in HCC.

Numerous key enzymes from the glycolysis pathway are involved in the carcinogenesis of HCC. The major distinction between HCC cells and normal hepatocytes is the difference in enzymes that catalyze the first step of glucose metabolism. In normal hepatocytes, this step is catalyzed by glucokinase, whereas this enzyme is lacking in HCC cells and is replaced by HK2 (80). The long non-coding RNA taurine upregulated gene 1 (TUG1) controls cell migration and glycolysis by regulating the $\mathrm{p} 21 / \mathrm{miR}-455-3 \mathrm{p}$ axis, which affects HK2 stability during translation but not transcription (81). In addition, miR-125a overexpression significantly decreases HK2 protein level in HCC cells, which indicates that miR-125a directly targets HK2 (82). In addition, overexpression of STAT3 upregulates HK2 mRNA and HK2 protein expression (83). Furthermore, hypomethylation in the HK2 promoter $\mathrm{CpG}$ island (CGI) $\mathrm{N}$-shore region increases HK2 expression, and hypermethylation in the HK2-CGI suppresses HK2 expression by inhibiting the interaction between a hypoxia response element and HIF-1 $\alpha$ (84).

PPP in breast cancer. G6PD is closely associated with molecular subtypes of breast cancer, and its upregulation is a negative prognostic factor in breast cancer $(85,86)$. It has been demonstrated that G6PD silencing increases the glycolytic flux, reduces lipid synthesis and increases glutamine uptake in breast cancer cells, whereas TKT silencing reduces glycolysis flux (31). Overexpression of NSD2 in breast cancer induces cancer resistance to tamoxifen by upregulating G6PD and HK2 expression, which enhances PPP flux (52). In addition, G6PD expression and activity are continuously unregulated in breast cancer cells, and it has been reported that G6PD inhibition leads to an increase in 5'-AMP-activated protein kinase (AMPK) signaling, a decrease in lipid biosynthesis and the inhibition of breast cancer cell growth and survival (21). Furthermore, TKT expression is associated with tumor size in the 4T1/BALB/c syngeneic model, and high TKT levels are associated with poor survival (87).

The YAP/TEAD/p65 axis upregulates HK2 transcription, which promotes breast cancer cell migration. This axis may therefore represent a potential therapeutic target for treatment of metastatic breast cancer (88). It has been reported that inhibition of hexokinase using 2-deoxyglucose induces chloroquine-resistance in breast cancer (89). In addition, metformin stimulates the glycolytic flux caused by starvation by interfering with HK2 activity (90). Furthermore, the AMPK-dependent phosphorylation of PFKFB3 substitutes oxidative respiration by glycolysis, which causes inhibition of cell death and of antitumor efficiency of the microtubule toxin in breast cancer cells (91). Sonic hedgehog phosphorylates PFKFB3 to promote glycolysis and proliferation of breast cancer cells, which is mediated by smoothened and p38/MK2 (92). In addition, the 6-phosphofructo-2-kinase/fructose-2,6-bisphosphatase 4 (PFKFB4) in breast cancer cells can phosphorylate the oncogenic steroid receptor coactivator-3, which rapidly increases its transcriptional activity and promotes the glucose flux switch towards purine synthesis (93). Furthermore, PKM2 is phosphorylated at tyrosine 105 and forms oncogenic dimers in breast cancer cells, whereas PKM2 is largely unphosphorylated and forms non-tumorigenic tetramers in non-transformed MCF10A cells (94). Moreover, the intragenic DNA methylation-mediated binding of the protein brother of regulator of imprinted sites on the replacement exon of PK is associated with cancer-specific splicing that promotes the Warburg effect and thus breast cancer progression (95).

PPP in lung cancer. G6PD is overexpressed in non-small cell lung carcinoma (NSCLC) (96), and the survival rate of patients with overexpressed G6PD protein is significantly poorer compared with those of patients with no G6PD overexpression (97). In addition, G6PD inhibition enhances lung cancer cell sensitivity to cisplatin by inducing oxidative stress (98). In addition, 6PGD promotes cisplatin resistance in lung cancer, through the decreased expression of miR-206 and miR-613 (23). G6PD and 6PGD may therefore represent potential novel targets to overcome cisplatin resistance. Furthermore, 6PGD is required for lung tumor cell migration in vitro through the promotion of c-Met phosphorylation at tyrosine residues (99). TKTL1 overexpression is an independent predictor of survival in NSCLC (100). Small interfering RNA-mediated silencing of 6PGD has been demonstrated to downregulate essential metabolic enzymes, including TKT, which leads to inhibition of lung cancer cell migration (101).

HK2 is essential for lung cancer cell growth in vitro and lung cancer tumorigenesis in vivo (102). It has been reported that EGFR signaling inhibition in NSCLC cells induces dramatic decrease in HK2 and PKM2 levels $(103,104)$. In addition, miR-214 downregulation inhibits HK2 expression and NSCLC cell proliferation (105). NAD(P)H:quinone oxidoreductase 1 increases HK2 gene expression, which enhances cellular glycometabolism and stimulates NSCLC cell proliferation (106). PFKFB1, 2, 3 and 4 mRNAs are overexpressed in human lung cancers compared with corresponding normal tissues (107). It has been demonstrated that miR-128 directly targets PFK liver type at the mRNA and protein levels in lung cancer cells by AKT phosphorylation inhibition (108). An increase in intracellular ROS leads to inhibition of the glycolytic enzyme PKM2 through oxidation of cysteine 358, which requires glucose transfer into the PPP; this phenomenon then stimulates ROS detoxification (68). All these enzymes may represent potential targets to develop novel strategies for diagnosis and treatment of lung cancer. 


\section{Perspectives}

The metabolic processes in cancer cells differ from those in normal cells. In malignancies, cancer cell proliferation is stimulated. Elevated PPP activity in cancer cells may distinguish cancer cells from normal cells, and the enzymes involved in PPP may therefore represent novel targets for diagnosis and treatment of various types of cancer. The present review demonstrated that cancer cells have acquired numerous mechanisms that circumvent PPP and glycolysis regulation. However, further investigation remainsessential to discoveradditional mechanisms and identify strategies for treating hyperactive PPP signaling in human cancers.

\section{Acknowledgements}

Not applicable.

\section{Funding}

The present study was supported by The National Natural Sciences Foundation of China (grant no. 81672685).

\section{Availability of data and materials}

Not applicable.

\section{Authors' contributions}

LJ drafted the manuscript. YZ revised the manuscript. All authors have read and approved the final version of this manuscript.

\section{Ethics approval and consent to participate}

Not applicable.

\section{Patient consent for publication}

Not applicable.

\section{Competing of interest}

The authors declare that they have no competing interest.

\section{References}

1. Vazquez A, Kamphorst JJ, Markert EK, Schug ZT, Tardito S and Gottlieb E: Cancer metabolism at a glance. J Cell Sci 129: 3367-3373, 2016.

2. Weber GF: Metabolism in cancer metastasis. Int J Cancer 138: 2061-2066, 2016.

3. Lu J, Tan M and Cai Q: The Warburg effect in tumor progression: Mitochondrial oxidative metabolism as an anti-metastasis mechanism. Cancer Lett 356: 156-164, 2015.

4. Vander Heiden MG, Cantley LC and Thompson CB: Understanding the Warburg effect: The metabolic requirements of cell proliferation. Science 324: 1029-1033, 2009.

5. Martinez-Outschoorn UE, Peiris-Pagés M, Pestell RG, Sotgia F and Lisanti MP: Cancer metabolism: A therapeutic perspective. Nat Rev Clin Oncol 14: 11-31, 2017.

6. Ramos-Martinez JI: The regulation of the pentose phosphate pathway: Remember Krebs. Arch Biochem Biophys 614: 50-52, 2017.
7. Patra KC and Hay N: The pentose phosphate pathway and cancer. Trends Biochem Sci 39: 347-354, 2014.

8. Kruger NJ and von Schaewen A: The oxidative pentose phosphate pathway: Structure and organisation. Curr Opin Plant Biol 6: 236-246, 2003.

9. Pavlova NN and Thompson CB: The emerging hallmarks of cancer metabolism. Cell Metab 23: 27-47, 2016.

10. Stincone A, Prigione A, Cramer T, Wamelink MM, Campbell K, Cheung E, Olin-Sandoval V, Grüning NM, Krüger A, Tauqeer Alam M, et al: The return of metabolism: Biochemistry and physiology of the pentose phosphate pathway. Biol Rev Camb Philos Soc 90: 927-963, 2015.

11. Zhang C, Zhang Z, Zhu Y and Qin S: Glucose-6-phosphate dehydrogenase: A biomarker and potential therapeutic target for cancer. Anticancer Agents Med Chem 14: 280-289, 2014.

12. Kathagen-Buhmann A, Schulte A, Weller J, Holz M, Herold-Mende C, Glass R and Lamszus K: Glycolysis and the pentose phosphate pathway are differentially associated with the dichotomous regulation of glioblastoma cell migration versus proliferation. Neuro Oncol 18: 1219-1229, 2016.

13. Ju HQ, Lu YX, Wu QN, Liu J, Zeng ZL, Mo HY, Chen Y, Tian T, Wang Y, Kang TB, et al: Disrupting G6PD-mediated Redox homeostasis enhances chemosensitivity in colorectal cancer. Oncogene 36: 6282-6292, 2017.

14. Au SW, Gover S, Lam VM and Adams MJ: Human glucose-6-phosphate dehydrogenase: The crystal structure reveals a structural NADP $(+)$ molecule and provides insights into enzyme deficiency. Structure 8: 293-303, 2000.

15. Cohen $\mathrm{P}$ and Rosemeyer MA: Subunit interactions of glucose-6-phosphate dehydrogenase from human erythrocytes. Eur J Biochem 8: 8-15, 1969.

16. Jiang P, Du W, Wang X, Mancuso A, Gao X, Wu M and Yang X: p53 regulates biosynthesis through direct inactivation of glucose-6-phosphate dehydrogenase. Nat Cell Biol 13: 310-316, 2011.

17. Ma X, Wang L, Huang, Li Y, Yang D, Li T, Li F, Sun L, Wei H, He K, et al: Polo-like kinase 1 coordinates biosynthesis during cell cycle progression by directly activating pentose phosphate pathway. Nat Commun 8: 1506, 2017.

18. Rao X, Duan X, Mao W, Li X, Li Z, Li Q, Zheng Z, Xu H, Chen M, Wang PG, et al: O-GlcNAcylation of G6PD promotes the pentose phosphate pathway and tumor growth. Nat Commun 6: 8468, 2015.

19. Thiepold AL, Lorenz NI, Foltyn M, Engel AL, Divé I, Urban H, Heller S, Bruns I, Hofmann U, Dröse S, et al: Mammalian target of rapamycin complex 1 activation sensitizes human glioma cells to hypoxia-induced cell death. Brain 140: 2623-2638, 2017.

20. Zhang X, Zhang X, Li Y, Shao Y, Xiao J, Zhu G and Li F: PAK4 regulates G6PD activity by p53 degradation involving colon cancer cell growth. Cell Death Dis 8: e2820, 2017.

21. Yang X, Peng X and Huang J: Inhibiting 6-phosphogluconate dehydrogenase selectively targets breast cancer through AMPK activation. Clin Transl Oncol 20: 1145-1152, 2018.

22. Bhanot H, Weisberg EL, Reddy MM, Nonami A, Neuberg D, Stone RM, Podar K, Salgia R, Griffin JD and Sattler M: Acute myeloid leukemia cells require 6-phosphogluconate dehydrogenase for cell growth and NADPH-dependent metabolic reprogramming. Oncotarget 8: 67639-67650, 2017.

23. Zheng W, Feng Q, Liu J, Guo Y, Gao L, Li R, Xu M, Yan G, Yin Z,Zhang S, et al: Inhibition of 6-phosphogluconate dehydrogenase reverses cisplatin resistance in ovarian and lung cancer. Front Pharmacol 8: 421, 2017.

24. Shan C, Elf S, Ji Q, Kang HB, Zhou L, Hitosugi T, Jin L, Lin R, Zhang L, Seo JH, et al: Lysine acetylation activates 6-phosphogluconate dehydrogenase to promote tumor growth. Mol Cell 55: $552-565,2014$.

25. Lin R, Elf S, Shan C, Kang HB, Ji Q, Zhou L, Hitosugi T, Zhang L, Zhang S, Seo JH, et al: 6-Phosphogluconate dehydrogenase links oxidative PPP, lipogenesis and tumour growth by inhibiting LKB1-AMPK signalling. Nat Cell Biol 17: 1484-1496, 2015.

26. Hitosugi T, Zhou L, Elf S, Fan J, Kang HB, Seo JH, Shan C, Dai Q, Zhang L, Xie J, et al: Phosphoglycerate mutase 1 coordinates glycolysis and biosynthesis to promote tumor growth. Cancer Cell 22: 585-600, 2012.

27. Yao P, Sun H, Xu C, Chen T, Zou B, Jiang P and Du W: Evidence for a direct cross-talk between malic enzyme and the pentose phosphate pathway via structural interactions. J Biol Chem 292: 17113-17120, 2017.

28. Qiu Z, Guo W, Wang Q, Chen Z, Huang S, Zhao F, Yao M, Zhao Y and He X: MicroRNA-124 reduces the pentose phosphate pathway and proliferation by targeting PRPS1 and RPIA mRNAs in human colorectal cancer cells. Gastroenterology 149: 1587-1598.e11, 2015. 
29. Chou YT, Jiang JK, Yang MH, Lu JW, Lin HK, Wang HD and Yuh $\mathrm{CH}$ : Identification of a noncanonical function for ribose-5-phosphate isomerase A promotes colorectal cancer formation by stabilizing and activating $\beta$-catenin via a novel C-terminal domain. PLoS Biol 16: e2003714, 2018.

30. Ciou SC, Chou YT, Liu YL, Nieh YC, Lu JW, Huang SF, Chou YT, Cheng LH, Lo JF, Chen MJ, et al: Ribose-5-phosphate isomerase A regulates hepatocarcinogenesis via PP2A and ERK signaling. Int J Cancer 137: 104-115, 2015.

31. Benito A, Polat IH, Noé V, Ciudad CJ, Marin S and Cascante M: Glucose-6-phosphate dehydrogenase and transketolase modulate breast cancer cell metabolic reprogramming and correlate with poor patient outcome. Oncotarget 8: 106693-106706, 2017.

32. Xu IM, Lai RK, Lin SH, Tse AP, Chiu DK, Koh HY, Law CT, Wong CM, Cai Z, Wong CC and Ng IO: Transketolase counteracts oxidative stress to drive cancer development. Proc Natl Acad Sci USA 113: E725-E734, 2016.

33. Kamenisch Y, Baban TSA, Schuller W, von Thaler AK, Sinnberg T, Metzler G, Bauer J, Schittek B, Garbe C, Rocken M and Berneburg M: UVA-irradiation induces melanoma invasion via the enhanced Warburg effect. J Invest Dermatol 136: 1866-1875, 2016.

34. Liu H, Huang D, McArthur D, Boros L, Nissen N and Heaney A: Fructose induces transketolase flux to promote pancreatic cancer growth. Cancer Res 70: 6368-6376, 2010.

35. Coy JF, Dressler D, Wilde J and Schubert P: Mutations in the transketolase-like gene TKTL1: Clinical implications for neurodegenerative diseases, diabetes and cancer. Clin Lab 51: 257-273, 2005

36. Coy JF, Dübel S, Kioschis P, Thomas K, Micklem G, Delius H and Poustka A: Molecular cloning of tissue-specific transcripts of a transketolase-related gene: Implications for the evolution of new vertebrate genes. Genomics 32: 309-316, 1996.

37. Langbein S, Zerilli M, Zur Hausen A, Staiger W, Rensch-Boschert K, Lukan N, Popa J, Ternullo MP, Steidler A, Weiss C, et al: Expression of transketolase TKTL1 predicts colon and urothelial cancer patient survival: Warburg effect reinterpreted. Br J Cancer 94: 578-585, 2006.

38. Lange CA, Tisch-Rottensteiner J, Böhringer D, Martin G, Schwartzkopff J and Auw-Haedrich C: Enhanced TKTL1 expression in malignant tumors of the ocular adnexa predicts clinical outcome. Ophthalmology 119: 1924-1929, 2012.

39. Lin CC, Chen LC, Tseng VS, Yan JJ, Lai WW, Su WP, Lin CH, Huang CY and Su WC: Malignant pleural effusion cells show aberrant glucose metabolism gene expression. Eur Respir J 37: $1453-1465,2011$

40. Xu X, Zur Hausen A, Coy JF and Löchelt M: Transketolase-like protein 1 (TKTL1) is required for rapid cell growth and full viability of human tumor cells. Int J Cancer 124: 1330-1337, 2009

41. Kočevar N, Odreman F, Vindigni A, Grazio SF and Komel R: Proteomic analysis of gastric cancer and immunoblot validation of potential biomarkers. World J Gastroenterol 18: 1216-1228, 2012.

42. Wang C, Guo K, Gao D, Kang X, Jiang K, Li Y, Sun L, Zhang S, Sun C, Liu X, et al: Identification of transaldolase as a nove serum biomarker for hepatocellular carcinoma metastasis using xenografted mouse model and clinic samples. Cancer Lett 313 154-166, 2011

43. Hsieh BS, Huang LW, Su SJ, Cheng HL, Hu YC, Hung TC and Chang KL: Combined arginine and ascorbic acid treatment induces apoptosis in the hepatoma cell line HA22T/VGH and changes in redox status involving the pentose phosphate pathway and reactive oxygen and nitrogen species. J Nutr Biochem 22 234-241, 2011

44. Li Z, Zhang B, Yao W, Zhang C, Wan L and Zhang Y: APC-Cdh1 regulates neuronal apoptosis through modulating glycolysis and pentose-phosphate pathway after oxygen-glucose deprivation and reperfusion. Cell Mol Neurobiol 39: 123-135, 2019.

45. Senyilmaz D and Teleman A: Chicken or the egg: Warburg effect and mitochondrial dysfunction. F1000Prime Rep 7: 41, 2015

46. Wilson JE: Isozymes of mammalian hexokinase: Structure, subcellular localization and metabolic function. J Exp Biol 206: 2049-2057, 2003.

47. Gu J, Singh A, Xue K, Mavis C, Barth M, Yanamadala V, Lenz P, Grau M, Lenz G, Czuczman MS and Hernandez-Ilizaliturri FJ: Up-regulation of hexokinase II contributes to rituximab-chemotherapy resistance and is a clinically relevant target for therapeutic development. Oncotarget 9: 4020-4033, 2017.

48. Patra KC, Wang Q, Bhaskar PT, Miller L, Wang Z, Wheaton W, Chandel N, Laakso M, Muller WJ, Allen EL, et al: Hexokinase 2 is required for tumor initiation and maintenance and its systemic deletion is therapeutic in mouse models of cancer. Cancer Cell 24: 213-228, 2013
49. An MX, Li S, Yao HB, Li C, Wang JM, Sun J, Li XY, Meng XN and Wang HQ: BAG3 directly stabilizes Hexokinase 2 mRNA and promotes aerobic glycolysis in pancreatic cancer cells. J Cell Biol 216: 4091-4105, 2017

50. Hoppe-Seyler K, Honegger A, Bossler F, Sponagel J, Bulkescher J, Lohrey C and Hoppe-Seyler F: Viral E6/E7 oncogene and cellular hexokinase 2 expression in HPV-positive cancer cell lines. Oncotarget 8: 106342-106351, 2017.

51. Ju HQ, Zhan G, Huang A, Sun Y, Wen S, Yang J, Lu WH, Xu RH, Li J, Li Y, et al: ITD mutation in FLT3 tyrosine kinase promotes Warburg effect and renders therapeutic sensitivity to glycolytic inhibition. Leukemia 31: 2143-2150, 2017.

52. Wang J, Duan Z, Nugent Z, Zou JX, Borowsky AD, Zhang Y, Tepper CG, Li JJ, Fiehn O, Xu J, et al: Reprogramming metabolism by histone methyltransferase NSD2 drives endocrine resistance via coordinated activation of pentose phosphate pathway enzymes. Cancer Lett 378: 69-79, 2016.

53. Ha JH, Radhakrishnan R, Jayaraman M, Yan M, Ward JD, Fung KM, Moxley K, Sood AK, Isidoro C, Mukherjee P, et al: LPA induces metabolic reprogramming in ovarian cancer via a pseudohypoxic response. Cancer Res 78: 1923-1934, 2018.

54. Moon JS, Kim HE, Koh E, Park SH, Jin WJ, Park BW, Park SW and Kim KS: Krüppel-like factor 4 (KLF4) activates the transcription of the gene for the platelet isoform of phosphofructokinase (PFKP) in breast cancer. J Biol Chem 286: 23808-23816, 2011.

55. Park YY, Kim SB, Han HD, Sohn BH, Kim JH, Liang J, Lu Y, Rodriguez-Aguayo C, Lopez-Berestein G, Mills GB, et al: Tat-activating regulatory DNA-binding protein regulates glycolysis in hepatocellular carcinoma by regulating the platelet isoform of phosphofructokinase through microRNA 520 Hepatology 58: 182-191, 2013.

56. Cabrera R, Baez M, Pereira HM, Caniuguir A, Garratt RC and Babul J: The crystal complex of phosphofructokinase-2 of Escherichia coli with fructose-6-phosphate: Kinetic and structural analysis of the allosteric ATP inhibition. J Biol Chem 286: 5774-5783, 2011.

57. Yi W, Clark PM, Mason DE, Keenan MC, Hill C, Goddard WA III, Peters EC, Driggers EM and Hsieh-Wilson LC: Phosphofructokinase 1 glycosylation regulates cell growth and metabolism. Science 337: 975-980, 2012.

58. Wang S, Peng Z, Wang S, Yang L, Chen Y, Kong X, Song S, Pei P, Tian C, Yan H, et al: KRAB-type zinc-finger proteins PITA and PISA specifically regulate p53-dependent glycolysis and mitochondrial respiration. Cell Res 28: 572-592, 2018.

59. Lee JH, Liu R, Li J, Zhang C, Wang Y, Cai Q, Qian X, Xia Y, Zheng Y, Piao Y, et al: Stabilization of phosphofructokinase 1 platelet isoform by AKT promotes tumorigenesis. Nat Commun 8: 949, 2017.

60. Wang H, Nicolay BN, Chick JM, Gao X, Geng Y, Ren H, Gao H, Yang G, Williams JA, Suski JM, et al: The metabolic function of cyclin D3-CDK6 kinase in cancer cell survival. Nature 546: 426-430, 2017.

61. Kim NH, Cha YH, Lee J, Lee SH, Yang JH, Yun JS, Cho ES, Zhang X, Nam M, Kim N, et al: Snail reprograms glucose metabolism by repressing phosphofructokinase PFKP allowing cancer cell survival under metabolic stress. Nat Commun 8: 14374, 2017.

62. Yamamoto T, Takano N, Ishiwata K, Ohmura M, Nagahata Y, Matsuura T, Kamata A, Sakamoto K, Nakanishi T, Kubo A, et al: Reduced methylation of PFKFB3 in cancer cells shunts glucose towards the pentose phosphate pathway. Nat Commun 5: 3480, 2014.

63. Gui DY, Lewis CA and Vander Heiden MG: Allosteric regulation of PKM2 allows cellular adaptation to different physiological states. Sci Signal 263: pe7, 2013.

64. Fukuda S, Miyata H, Miyazaki Y, Makino T, Takahashi T, Kurokawa Y, Yamasaki M, Nakajima K, Takiguchi S, Mori M and Doki Y: Pyruvate kinase M2 modulates esophageal squamous cell carcinoma chemotherapy response by regulating the pentose phosphate pathway. Ann Surg Oncol 22 (Suppl 3): S1461-S1468, 2015.

65. Israelsen WJ, Dayton TL, Davidson SM, Fiske BP, Hosios AM, Bellinger G, Li J, Yu Y, Sasaki M, Horner JW, et al: PKM2 isoform-specific deletion reveals a differential requirement for pyruvate kinase in tumor cells. Cell 155: 397-409, 2013.

66. Keller KE, Tan IS and Lee YS: SAICAR stimulates pyruvate kinase isoform M2 and promotes cancer cell survival in glucose-limited conditions. Science 338: 1069-1072, 2012.

67. Yang W, Xia Y, Hawke D, Li X, Liang J, Xing D, Aldape K, Hunter T, Alfred Yung WK and Lu Z: PKM2 phosphorylates histone $\mathrm{H} 3$ and promotes gene transcription and tumorigenesis. Cell 150: 685-696, 2012. 
68. Anastasiou D, Poulogiannis G, Asara JM, Boxer MB, Jiang JK Shen M, Bellinger G, Sasaki AT, Locasale JW, Auld DS, et al: Inhibition of pyruvate kinase $\mathrm{M} 2$ by reactive oxygen species contributes to cellular antioxidant responses. Science 334: 1278-1283, 2011.

69. Luo W, Hu H, Chang R, Zhong J, Knabel M, O'Meally R, Cole RN, Pandey A and Semenza GL: Pyruvate kinase M2 is a PHD3-stimulated coactivator for hypoxia-inducible factor 1 . Cell 145: 732-744, 2011.

70. Chaneton B, Hillmann P,Zheng L, Martin ACL, Maddocks ODK Chokkathukalam A, Coyle JE, Jankevics A, Holding FP, Vousden $\mathrm{KH}$, et al: Serine is a natural ligand and allosteric activator of pyruvate kinase M2. Nature 491: 458-462, 2012.

71. Goh GB, Li JW, Chang PE, Chow KY and Tan CK: Deciphering the epidemiology of hepatocellular carcinoma through the passage of time: A study of 1,401 patients across 3 decades. Hepatol Commun 1: 564-571, 2017.

72. Busby J, Mills K, Zhang S, Liberante F and Cardwell C: Postdiagnostic Calcium channel blocker use and breast cancer mortality: A population-based cohort study. Epidemiology 29: 407-413, 2018

73. Nanavaty P, Alvarez MS and Alberts WM: Lung cancer screening: Advantages, controversies, and applications. Cancer Control 21: 9-14, 2014.

74. Lu M, Lu L, Dong Q, Yu G, Chen J, Qin L, Wang L, Zhu W and Jia H: Elevated G6PD expression contributes to migration and invasion of hepatocellular carcinoma cells by inducing epithelial-mesenchymal transition. Acta Biochim Biophys Sin (Shanghai) 50: 370-380, 2018

75. Kowalik M, Guzzo G, Morandi A,Perra A, Menegon S, Masgras I, Trevisan E, Angioni MM,Fornari F, Quagliata L, et al: Metabolic reprogramming identifies the most aggressive lesions at early phases of hepatic carcinogenesis. Oncotarget 7: 32375-32393, 2016.

76. Kong DH, Li S, Du ZX, Liu C, Liu BQ, Li C, Zong ZH and Wang HQ: BAG3 elevation inhibits cell proliferation via direct interaction with G6PD in hepatocellular carcinomas. Oncotarget 7: 700-711, 2016.

77. Hong X, Song R, Song H, Zheng T, Wang J, Liang Y, Qi S, Lu Z, Song $\mathrm{X}$, Jiang $\mathrm{H}$, et al: PTEN antagonises Tcl1/hnRNPK-mediated G6PD pre-mRNA splicing which contributes to hepatocarcinogenesis. Gut 63: 1635-1647, 2014

78. Maruyama H, Kleeff J, Wildi S, Friess H, Büchler MW, Israel MA and Korc M: Id-1 and Id-2 are overexpressed in pancreatic cancer and in dysplastic lesions in chronic pancreatitis. Am J Pathol 155: 815-822, 1999.

79. Yin X, Tang B, Li JH, Wang Y, Zhang L, Xie XY, Zhang BH, Qiu SJ, Wu WZ and Ren ZG: ID1 promotes hepatocellular carcinoma proliferation and confers chemoresistance to oxaliplatin by activating pentose phosphate pathway. J Exp Clin Cancer Res 36: 166, 2017.

80. DeWaal D, Nogueira V, Terry AR, Patra KC, Jeon SM, Guzman G, Au J, Long CP, Antoniewicz MR and Hay N: Hexokinase-2 depletion inhibits glycolysis and induces oxidative phosphorylation in hepatocellular carcinoma and sensitizes to metformin. Nat Commun 9: 446, 2018.

81. Lin YH, Wu MH, Huang YH, Yeh CT, Cheng ML, Chi HC, Tsai CY, Chung IH, Chen CY and Lin KH: Taurine up-regulated gene 1 functions as a master regulator to coordinate glycolysis and metastasis in hepatocellular carcinoma. Hepatology 67: 188-203, 2018

82.Jin F, Wang Y, Zhu Y, Li S, Liu Y, Chen C, Wang X, Zen K and $\mathrm{Li} \mathrm{L}$ : The miR-125a/HK2 axis regulates cancer cell energy metabolism reprogramming in hepatocellular carcinoma. Sci Rep 7: 3089, 2017.

83. Li M, Jin R, Wang W, Zhang T, Sang J, Li N, Han Q, Zhao W, $\mathrm{Li} \mathrm{C}$ and Liu Z: STAT3 regulates glycolysis via targeting hexokinase 2 in hepatocellular carcinoma cells. Oncotarget 8 : 24777-24784, 2017.

84. Lee H, Kim H, Son T, Jeong Y, Kim SU, Dong SM, Park YN, Lee JD, Lee JM and Park JH: Regulation of HK2 expression through alterations in $\mathrm{CpG}$ methylation of the HK2 promoter during progression of hepatocellular carcinoma. Oncotarget 7: 41798-41810, 2016.

85. Dong T, Kang X, Liu Z, Zhao S, Ma W, Xuan Q, Liu H, Wang Z and Zhang Q: Altered glycometabolism affects both clinical features and prognosis of triple-negative and neoadjuvant chemotherapy-treated breast cancer. Tumour Biol 37: 8159-8168, 2016.
86. Pu H, Zhang Q, Zhao C, Shi L, Wang Y, Wang J and Zhang M Overexpression of G6PD is associated with high risks of recurrent metastasis and poor progression-free survival in primary breast carcinoma. World J Surg Oncol 13: 323, 2015.

87. Tseng CW, Kuo WH, Chan SH, Chan HL, Chang KJ and Wang LH: Transketolase regulates the metabolic switch to control breast cancer cell metastasis via the $\alpha$-ketoglutarate signaling pathway. Cancer Res 78: 2799-2812, 2018

88. Gao Y, Yang Y, Yuan F, Huang J, Xu W, Mao B, Yuan Z and $\mathrm{Bi}$ W: TNF $\alpha-Y A P / p 65-H K 2$ axis mediates breast cancer cell migration. Oncogenesis 6: e383, 2017

89. Gallagher LE, Radhi OA, Abdullah MO, McCluskey AG, Boyd M and Chan EYW: Lysosomotropism depends on glucose: A chloroquine resistance mechanism. Cell Death Dis 8: e3014, 2017.

90. Marini C, Bianchi G, Buschiazzo A, Ravera S, Martella R, Bottoni G, Petretto A, Emionite L, Monteverde E, Capitanio S, et al: Divergent targets of glycolysis and oxidative phosphorylation result in additive effects of metformin and starvation in colon and breast cancer. Sci Rep 6: 19569, 2016.

91. Doménech E, Maestre C, Esteban-Martínez L, Partida D, Pascual R, Fernández-Miranda G, Seco E, Campos-Olivas R, Pérez M, Megias D, et al: AMPK and PFKFB3 mediate glycolysis and survival in response to mitophagy during mitotic arrest. Nat Cell Biol 17: 1304-1316, 2015.

92. Ge X, Lyu P, Gu Y, Li L, Li J, Wang Y, Zhang L, Fu C and Cao Z: Sonic hedgehog stimulates glycolysis and proliferation of breast cancer cells: Modulation of PFKFB3 activation. Biochem Biophys Res Commun 464: 862-868, 2015.

93. Dasgupta S, Rajapakshe K, Zhu B, Nikolai BC, Yi P, Putluri N, Choi JM, Jung SY, Coarfa C, Westbrook TF, et al: Metabolic enzyme PFKFB4 activates transcriptional coactivator SRC-3 to drive breast cancer. Nature 556: 249-254, 2018.

94. Zhou Z, Li M, Zhang L, Zhao H, Şahin Ö, Chen J, Zhao JJ, Songyang Z and Yu D: Oncogenic kinase-induced PKM2 tyrosine 105 phosphorylation converts non-oncogenic PKM2 to a tumor promoter and induces cancer stem-like cells. Cancer Res 78: 2248-2261, 2018

95. Singh S, Narayanan S, Biswas K, Gupta A, Ahuja N, Yadav S, Panday RK, Samaiya A, Sharan SK and Shukla S: Intragenic DNA methylation and BORIS-mediated cancer-specific splicing contribute to the Warburg effect. Proc Natl Acad Sci USA 114: 11440-11445, 2017.

96. Giatromanolaki A, Sivridis E, Arelaki S and Koukourakis M Expression of enzymes related to glucose metabolism in non-small cell lung cancer and prognosis. Exp Lung Res 43: $167-174,2017$.

97. Nagashio R, Oikawa S, Yanagita K, Hagiuda D, Kuchitsu Y, Igawa S, Naoki K, Satoh Y, Ichinoe M, Murakumo Y, et al: Prognostic significance of G6PD expression and localization in lung adenocarcinoma. Biochim Biophys Acta Proteins Proteom 1867: 38-46, 2019.

98. Hong W, Cai P, Xu C, Cao D, Yu W, Zhao Z, Huang M and Jin J: Inhibition of Glucose-6-phosphate dehydrogenase reverses cisplatin resistance in lung cancer cells via the redox system. Front Pharmacol 9: 43, 2018.

99. Chan B, VanderLaan P and Sukhatme VP: 6-Phosphogluconate dehydrogenase regulates tumor cell migration in vitro by regulating receptor tyrosine kinase c-Met. Biochem Biophys Res Commun 439: 247-251, 2013.

100. Kayser G, Sienel W, Kubitz B, Mattern D, Stickeler E, Passlick B, Werner M and Zur Hausen A: Poor outcome in primary non-small cell lung cancers is predicted by transketolase TKTL1 expression. Pathology 43: 719-724, 2011.

101. $\mathrm{Lu} \mathrm{H}$ and Zhu H: Effect of siRNA-mediated gene silencing of transketolase on A549 lung cancer cells. Oncol Lett 14: 5906-5912, 2017.

102. Wang H, Wang L, Zhang Y, Wang J, Deng Y and Lin D: Inhibition of glycolytic enzyme hexokinase II (HK2) suppresses lung tumor growth. Cancer Cell Int 16: 9, 2016.

103. De Rosa V, Iommelli F, Monti M, Fonti R, Votta G, Stoppelli MP and Del Vecchio S: Reversal of Warburg effect and reactivation of oxidative phosphorylation by differential inhibition of EGFR signaling pathways in non-small cell lung cancer. Clin Cancer Res 21: 5110-5120, 2015

104. Su H, Bodenstein C, Dumont RA, Seimbille Y, Dubinett S, Phelps ME, Herschman H, Czernin J and Weber W: Monitoring tumor glucose utilization by positron emission tomography for the prediction of treatment response to epidermal growth factor receptor kinase inhibitors. Clin Cancer Res 12: 5659-5667, 2006. 
105. Zhang K, Zhang M, Jiang H, Liu F, Liu H and Li Y: Down-regulation of miR-214 inhibits proliferation and glycolysis in non-small-cell lung cancer cells via down-regulating the expression of hexokinase 2 and pyruvate kinase isozyme M2. Biomed Pharmacother 105: 545-552, 2018.

106. Cheng X, Liu F, Liu H, Wang G and Hao H: Enhanced glycometabolism as a mechanism of NQO1 potentiated growth of NSCLC revealed by metabolomic profiling. Biochem Biophys Res Commun 496: 31-36, 2018.
107. Minchenko OH, Ogura T, Opentanova IL, Minchenko DO, Ochiai A, Caro J, Komisarenko SV and Esumi H: 6-Phosphofructo-2-kinase/fructose-2,6-bisphosphatase gene family overexpression in human lung tumor. Ukr Biokhim Zh (1999) 77: 46-50, 2005

108. Yang J, Li J, Le Y, Zhou C, Zhang S and Gong Z: PFKL/miR-128 axis regulates glycolysis by inhibiting AKT phosphorylation and predicts poor survival in lung cancer. Am J Cancer Res 6: 473-485, 2016. 